\title{
Physical characterization of biomedical magnetic nanoparticles using multi-detector centrifugal field-flow fractionation
}

Amani Remmo, Physikalisch-Technische Bundesanstalt (8.23 Metrology for Magnetic Nanoparticles), Berlin, Germany, amani.remmo@ptb.de

Norbert Löwa, Physikalisch-Technische Bundesanstalt (8.23 Metrology for Magnetic Nanoparticles), Berlin, Germany, norbert.loewa@ptb.de

Julija Peter, TU Berlin (Institut für Strömungsmechanik und Technische Akustik), Straße des 17. Juni 135, 10623 Berlin, Germany, julija.peter@tu-berlin.de

Frank Wiekhorst, Physikalisch-Technische Bundesanstalt (8.23 Metrology for Magnetic Nanoparticles), Berlin, Germany, frank.wiekhorst@ptb.de

\section{Introduction}

The unique magnetic properties of magnetic nanoparticles (MNP) combined with their small size have already led to numerous medical applications. They are used in in-vitro diagnostics for magnetic separation, as contrast agents in invivo imaging diagnostics or in tumor therapy. For each of these applications, MNPs with specific structural and magnetic properties must be provided. Accurate determination of their magnetic properties is a key requirement enquired by users, that is impeded by the ever-present distribution of MNP sizes. For this purpose, we developed and validated a method to separate the MNP distribution with respect to mass followed by direct characterization of the fraction populations using complementary detectors.

\section{Methods}

The mass sensitive separation of MNP was performed using the commercial centrifugal field-flow fractionation system (CFFF, Postnova Analytics GmbH). The downstream detector array consisted of a UV detector for concentration determination, a MALS detector for core size determination, a DLS detector for hydrodynamic size determination, and a magnetic particle spectrometer for magnetic characterization. The detector array was calibrated using a suitable MNP reference sample (SHP-25-50, Ocean Nanotech). Subsequently, the fractionation process was optimized with regard to retention ratio, selectivity, and recovery.

\section{Results}

It was shown that using the multi-detector CFFF method, MNP can be accurately and reproducibly fractionated into their component populations and their associated size and size distribution as well as the associated magnetic behavior can be characterized, regardless of whether they are monomodal or mulit-modal. Moreover, the results show that the method is suitable to provide information on the suitability of MNP for novel magnetic particle imaging. Since the MNP are fractionated in the liquid stream and do not come into contact with the separation channel, significantly higher recoveries were obtained compared to similar methods (e.g. asymmetric field flow fractionation). This was demonstrated on the commercial MNP system Resovist ${ }^{\circledR}$ (MRI liver contrast agent).

\section{Conclusion}

We showed that the new method for mass sensitive fractionation of MNP and subsequent direct physical characterization facilitates the automated, reliable, and reproducible determination of critical quality attributes of MNP. With the new method, these quality attributes can be accessed even faster and thus support the research and development of biomedical MNP applications. 


\section{In vitro biocompatibility evaluation of a magnetic nanoporous silica nanoparticle}

Ronja Storjohann, Department of Pharmacology, Toxicology and Pharmacy, University of Veterinary Medicine Hannover, Foundation, Hannover, Germany, Ronja.Storjohann@tiho-hannover.de

Hilke Oltmanns, Department of Pharmacology, Toxicology and Pharmacy, University of Veterinary Medicine Hannover, Foundation, Hannover, Germany

Timo Herrmann, Institute of Inorganic Chemistry, Leibniz University Hannover, Hannover, Germany

Peter Behrens, Institute of Inorganic Chemistry, Leibniz University Hannover, Hannover, Germany

Nina Angrisani, NIFE-Lower Saxony Centre for Biomedical Engineering, Implant Research and Development, Clinic for Orthopedic Surgery, Hannover Medical School, Hannover, Germany

Janine Reifenrath, NIFE-Lower Saxony Centre for Biomedical Engineering, Implant Research and Development, Clinic for Orthopedic Surgery, Hannover Medical School, Hannover, Germany

Jessica Meißner, Department of Pharmacology, Toxicology and Pharmacy, University of Veterinary Medicine Hannover, Foundation, Hannover, Germany

\section{Introduction}

Implant-associated infections are a widespread problem that often causes loosening of the implants and may necessitate revision surgeries. Therefore, magnetic nanoporous silica nanoparticles (NP) were constituted for targeted therapy and functionalized with a $10 \mathrm{kDa}$ poly(ethylene glycol)-silane. Biocompatibility was tested in different cell lines as well as in primary murine immune cells regarding the effect on cell proliferation and viability.

\section{Methods}

Subconfluent mouse fibroblast cell line L929 was treated with different amounts of NP $(15 \mu \mathrm{g} / \mathrm{mL}, 50 \mu \mathrm{g} / \mathrm{mL}$ and $150 \mu \mathrm{g} / \mathrm{mL}$ ) and stained with crystal violet assay (CV) after 24 and 48 hours. CellTiter $96^{\circledR}$ MTS was performed similarly on confluent plates. The humane hepatoma cell line HepG2 was analyzed with CV and MTS after 24 and 72 hours of treatment. Additionally, primary murine cells (dendritic cells and macrophages) were generated from male BALB/c mice. Immune cells were differentiated from bone marrow derived cells to study their viability in a comparable manner. All experiments included an untreated control, a lipopolysaccharide stimulated control and a control treated with dimethyl sulfoxide.

\section{Results}

L929 and HepG2 showed no effect of NP treatment on the cellular mass over the different incubation periods. Furthermore, there was no impact on the viability observed in both cell lines. After a 24 hour lasting treatment murine dendritic cells showed an increased viability, which correlates positively with the NP concentration in comparison to the untreated control. Viability of murine macrophages was not influenced by the NP over 24 and 48 hours.

\section{Conclusion}

This study reveals a good biocompatibility of magnetic NP regarding their effect on viability and proliferation in different cell types. However, further investigation is required for a profound understanding of ongoing mechanism, especially in immune cells and their cytokine secretion. 


\section{Visualization of SPIONs-loaded Monocytes as Inflammatory Sensors using Magnetic Particle Imaging}

Zuzana Penxová (Institute of Medical Engineering, University of Lübeck), Lübeck, Germany, zuzana.penxova@student.uni-luebeck.de

Kerstin Lüdtke-Buzug (Institute of Medical Engineering, University of Lübeck), Lübeck, Germany, luedtkebuzug@imt.uni-luebeck.de

Ralph Pries (Otorhinolaryngology, University Medical Center Schleswig-Holstein), Lübeck, Germany, Ralph.Pries@uksh.de

Christina Polasky (Otorhinolaryngology, University Medical Center Schleswig-Holstein), Lübeck, Germany, Christina.Polasky@uksh.de

Kristin Loyal (Otorhinolaryngology, University Medical Center Schleswig-Holstein), Lübeck, Germany, Kristin.Loyal@uksh.de

\section{Introduction}

Magnetic Particle Imaging (MPI) as a new promising functional imaging method is able to acquire images with high spatial and temporal resolution. Thereby, MPI offers many new possibilities for early diagnostics in the frame of individualized and precision medicine.

The magnetic nanoparticles as a tracer for MPI are usually recognized quickly by the immune system. Further functionalization and redesign of nanoparticles are necessary to allow tissue-/disease-specific imaging. Analog to PET/SPECT which uses the radioactively labeled glucose accumulating due to Warburg effect in the tumor tissue, development of similar concepts for MPI is of great interest.

This work aims to prove the concept of visualization of Superparamagnetic Iron Oxide Nanoparticles (SPIONs)-loaded monocytes as inflammatory sensors using MPI.

\section{Methods}

The approach followed here was a preparation of individualized biosensors. The body's natural cellular mechanisms are used to target the specific tissue. In the presented study, immune cells (monocytes) have been loaded with SPIONs.

Previously, the SPIONs were synthesized and post-processed to facilitate in-vitro application. The three types of applied SPIONs consisted of a non-magnetic dextran (MW 10, 20, $70 \mathrm{kDa})$ shell and a magnetite $\left(\mathrm{Fe}_{3} \mathrm{O}_{4}\right)$ core. The dextran coat of SPIONs was fluorescence-labeled with Fluorescein Isothiocyanate (FITC) to allow later detection via flow cytometry. The SPIONs-incorporation was realized by phagocytosis.

\section{Results}

The obtained results represent the interplay between incorporation of desired high concentration of SPIONs producing a strong magnetic signal for MPI on one side and viability of monocytes loaded with SPIONs on the other side.

\section{Conclusion}

In the frame of individualized medicine, human monocytes loaded with SPIONs could after further differentiation act as inflammation biosensors and probably enable a reliable detection of inflammation inside the body using MPI. 


\section{A multi-purpose phantom kit for magnetic particle imaging}

Norbert Löwa, Physikalisch-Technische Bundesanstalt (8.23 Metrology for Magnetic Nanoparticles), Berlin, Germany, norbert.loewa@ptb.de

Rebecca Hoffmann, TU Ilmenau (Fakultät für Informatik und Automatisierung, Institut für Biomedizinische Technik und Informatik), Ilmenau, Germany, becky.hoffmann@live.de

Dirk Gutkelch, Physikalisch-Technische Bundesanstalt (8.23 Metrology for Magnetic Nanoparticles), Berlin, Germany, dirk.gutkelch@ptb.de

Olaf Kosch, Physikalisch-Technische Bundesanstalt (8.23 Metrology for Magnetic Nanoparticles), Berlin, Germany, olaf.kosch@ptb.de

Silvio Dutz, TU Ilmenau (Fakultät für Informatik und Automatisierung, Institut für Biomedizinische Technik und Informatik), Ilmenau, Germany, silvio.dutz@tu-ilmenau.de

Frank Wiekhorst, Physikalisch-Technische Bundesanstalt (8.23 Metrology for Magnetic Nanoparticles), Berlin, Germany, frank.wiekhorst@ptb.de

\section{Introduction}

Phantoms are essential tools for the development and characterization of imaging techniques. They represent defined shapes and properties to investigate image resolution, sensitivity, or geometric distortions. Furthermore, they are used for training purposes to simulate application scenarios. Likewise, phantoms are mandatory for Magnetic Particle Imaging (MPI), an imaging technique that is capable of quantitatively imaging the spatial distribution of magnetic nanoparticles (MNP). Currently, the potential of MPI is being explored in relevant application areas through intensive research and development work, mainly on purpose-built phantoms, since MPI phantoms are commercially not available, so far. In this work a multi-purpose phantom kit was developed and validated considering specific requirements of MPI.

\section{Methods}

The basic concept of the phantom kit consists of a flexible modular structure composed of voxel-sized building blocks. The individual building blocks are made either of non-magnetic plastic or of novel plastic-MNP composite. The setup is designed to allow any magnetic structure $\left(>9 \mathrm{~mm}^{\wedge} 3\right)$ to be assembled in a reproducible and defined manner. To produce the individual magnetic building blocks, a special procedure was developed to homogeneously embed MNP in photopolymer resins. The photopolymer was then processed in a Digital Light Processing 3D printing system. The magnetic properties of each voxel-sized part were determined using high-sensitivity magnetic particle spectroscopy which is considered as 0-dimensional MPI. Finally, different application scenarios were built and mapped with the help of an MPI scanner (MPI 25/50FF, Bruker BioSpin) to demonstrate the capability and flexibility of the novel phantom kit.

\section{Results}

Characterization of the individual 3D-printed phantom kit constituents revealed a relative variation of less than $2 \%$ in the MPS signal amplitude, reflecting the high reproducibility of the manufacturing process. This was underpinned by an equally low variation in the geometric dimensions $(<2 \%)$. Since the magnetic properties of each building block can be acquired prior to a phantom experiment, a voxel-wise calibrated phantom can be created. Finally, the suitability of the MPI phantom kit was successfully demonstrated in various application scenarios (spatial resolution, imaging accuracy and quantification accuracy).

\section{Conclusion}

This work shows the development and validation of an MPI phantom kit considering specific requirements for MPI. It was shown that the developed MPI phantom kit can be used to study various issues in MPI, from spatial resolution to image accuracy (including anatomical replications) or to evaluate the quantification accuracy. In the future, the provision of such reference phantoms should enable direct comparison of existing scanners, leading to harmonization of measurement equipment for the MPI community. 


\section{Optimized Coil Configurations for Improved Magnetorelaxometry Imaging}

Peter Schier, Institute of Electrical and Biomedical Engineering, UMIT - Private University for Health Sciences, Medical Informatics and Technology, Hall in Tirol, Austria, peter.schier@umit-tirol.at

Maik Liebl, Physikalisch Technische Bundesanstalt (PTB), Braunschweig, Germany, maik.liebl@ptb.de

Frank Wiekhorst, Physikalisch Technische Bundesanstalt (PTB), Berlin, Germany, frank.wiekhorst@ptb.de

Daniel Baumgarten, Institute of Electrical and Biomedical Engineering, UMIT - Private University for Health Sciences, Medical Informatics and Technology, Hall in Tirol, Austria, daniel.baumgarten@umit-tirol.at

\section{Introduction}

Magnetorelaxometry imaging (MRXI) is an experimental imaging technique allowing for the non-invasive, quantitative reconstruction of magnetic nanoparticle (MNP) ensembles inside the body. This information is mandatory to ensure the safety and efficiency of several novel MNP-based therapies such as magnetic hyperthermia and magnetic drug targeting. However, an improvident choice of excitation coil configurations worsens the reconstruction accuracies and necessitates increased numbers of excitation sequences, hence prolonging the data acquisition time. Thus, we aim for optimizing MRXI coil configurations to enable the most accurate reconstructions for a given number of coils possible. The improved imaging quality of the optimized setups shall be validated through practical experiments.

\section{Methods}

MRXI involves solving a linear inverse problem. We optimize 32 coil positions of both a cuboidal and a cylindrical MRXI setup by minimizing the condition number of their corresponding system matrices, respectively. The optimization is performed using a global tabu search and a subsequent local gradient-based approach, both of which select coil positions and radii optimal with respect to the condition number. The resulting MRXI setups were built and tested at PTB Berlin using their 304 SQUID vector magnetometer system for recording the measurements. The imaging results of several different MNP phantoms are compared to the reconstructions of standard coil configurations.

\section{Results}

Each phantom of both setups has been reconstructed more accurately using the optimized coil configurations compared to the standard configurations.

\section{Conclusion}

It is possible to reconstruct MNP distributions more accurately and/or reduce the required data acquisition time for MRXI by following the proposed optimization approach for the coil configurations. Moreover, this optimization is not limited only to coil configurations, but can easily be extended to other crucial MRXI design parameters, such as sensor positions and orientations as well as excitation coil currents. 